\title{
Effect of Electron Correlation in the Decomposition of Core Level Binding Energy Shifts into Initial and Final State Contributions ${ }^{\dagger}$
}

\author{
Marc Figueras, Carmen Sousa, Francesc Illas*
}

Departament de Ciència de Materials i Química Física \& Institut de Química Teòrica i Computacional (IQTCUB), Universitat de Barcelona, c/ Martí i Franquès 1, 08028 Barcelona, Catalunya, Spain

\begin{abstract}
The influence of electron correlation into the decomposition of core level binding energy shifts, measured by X-ray photoelectron spectroscopy (XPS), into initial and final effects is analysed for a series of molecules where these effects are noticeable. Moreover, the series of molecules is chosen in such a way that electron delocalization and increasing number of electrons may provide a large screening of the core hole. A detailed analysis shows that the Hartree-Fock decomposition is biased whereas a physically meaningful decomposition is obtained when electron correlation effects are taken into account. The results show that in this case, trends in core level binding energy shifts are driven by initial state effects thus providing further support to the use of these observable quantities to interpret changes in the chemical bond in the neutral molecule rather than on the core ionized cation. Consequences for the theoretical interpretation of XPS data in materials and surface science are discussed.
\end{abstract}

e-mail: francesc.illas@ub.edu; Fax:+34-93-402-1231; Tel: +34-93-402-1229

$\dagger$ Electronic supplementary information (ESI) available: See DOI: 


\section{Introduction}

Photoelectron spectroscopy ${ }^{1}$ measures the kinetic energy of electrons ejected from a sample upon radiation and, through the well-known Einstein equation for the photoelectric effect, provides a measure of electron binding energies (BEs). Depending on the energy (frequency) of the incoming radiation - ultraviolet (UV) or X-Ray — valence or core electrons are ionized, and the resulting technique is usually referred to as ultraviolet photoelectron spectroscopy (UPS) or X-ray photoelectron spectroscopy (XPS). In particular, XPS is a powerful analytical technique broadly used in surface and materials science ${ }^{2}$ because the core level BEs (CLBEs) are unique for each chemical element. ${ }^{3,4}$ Even more, for a given element CLBEs may exhibit some shifts, hereafter denoted as $\triangle$ CLBEs, which are directly related to the chemical environment of the core ionized atom. ${ }^{5-7}$

One of the obvious applications of measured $\triangle$ CLBEs is establishing the oxidation state of a given element in different materials, e.g. $\mathrm{Fe}^{2+}$ or $\mathrm{Fe}^{3+}$ in $\mathrm{FeO}$ and $\mathrm{Fe}_{2} \mathrm{O}_{3}$, respectively. ${ }^{8}$ Here, there is a quite simple rule of thumb, the higher the oxidation state, the higher the CLBE simply because, due to the increased effect of the nuclear charge resulting from the electron removal, core levels in cations are shifted to higher energies and, hence, exhibit positive $\triangle$ CLBEs. The opposite holds for anions where the increased electron density for the same nuclear charge leads to smaller CLBEs. This argument is broadly used, and abused, to correlate measured $\triangle$ CLBEs to the net charge of atoms in the material before ionization. In fact, even if charge transfer is often one of the effects dominating $\triangle$ CLBEs, it is by no means the only one, as discussed in detail by Bagus and co-workers. ${ }^{9}$ In particular, hybridization and coordination have been described as mechanisms contributing to the measured $\triangle$ CLBEs. Nevertheless, the discussion above regarding the oxidation state of a given atom in a given chemical environment, strongly suggests that $\triangle$ CLBEs are already present in the neutral, non-ionized, sample and are usually referred to as initial state (IS) effects although, for a quantitative assessment, further analyses are required. The separation of initial and final state contributions to CLBEs is routinely used in the interpretation of $\mathrm{XPS}^{6,9}$ and its rigorous definition is recalled below. Here we just point out that XPS measurements provide information about final state binding energies only. Hence, the separation between initial and final state is just a theoretical construct for interpretative purposes. The main idea is that initial state binding energies contain the information about the chemical environment of the atom where core ionization will take place. More often, core level shifts are dominated by initial state effects which provides support to this separation and reinforces the interpretations. ${ }^{6}$

For a given set of materials containing a common element, it is possible to investigate to which extent IS effects dominate the observed $\triangle$ CLBEs using theoretical concepts and tools. ${ }^{5,6,9}$ For a generic core level, CLBEs can be predicted by the difference in total energy of the core-ionized and neutral system as in Eq. (1) 
Note that within the definition in Eq. (1) CLBEs are all positive as they correspond to the ionization energies. The accuracy of the computed value, defined with respect to the experimental value, depends on the method used to estimate the total energy. A recent survey analysed 185 CLBEs for atoms B to F of 68 molecules as computed by the Hartree-Fock (HF) and two density functional theory (DFT) based methods $;^{10}$ the latter include one GGA $(\mathrm{PBE})^{11}$ and one meta-GGA (revTPSS) ${ }^{12}$ exchange-correlation functional. The accuracy of HF and revTPSS was found to be similar with a mean absolute error below $0.3 \mathrm{eV}$ for the CLBEs which go to 0.11 and $0.05 \mathrm{eV}$ when relativistic effects are taken into account, thus reaching quantitative predictive power. Note in passing by that in the case of HF and DFT methods, each of the energy values in Eq. (1) is obtained from a self-consistent-field (SCF) calculation and the CLBEs thus computed are referred to as $\triangle \mathrm{SCF}$.

The CLBEs computed from Eq. (1) include all possible effects and, hence, do not allow for a separation of initial and final state (FS) effects. From a conceptual point of view, IS effects can be approached also by a total energy difference as in Eq. (1) but in such a way that the wave function or electron density of the neutral molecule is used to compute the energy of the core ionized molecule. ${ }^{5-7,9}$ In the framework of HF or DFT approaches, this means simply using the corresponding HF or Kohn-Sham molecular orbitals of the neutral molecule to compute the energy of the cation and the resulting approach is referred to as Frozen Orbital (FO); the FO computed CLBE is obtained from Eq. (2)

$$
\mathrm{CLBE}^{\mathrm{FO}}=\mathrm{E}^{\mathrm{FO}} \text { (core-ionized state)-E (neutral state) }
$$

The difference between CLBE and $\mathrm{CLBE}^{\mathrm{FO}}$ defines the FS effects, hereafter denoted as $\mathrm{E}_{\mathrm{R}}$ and, with the present definition, being always a negative value. In the case of CLBEs computed from the HF method, $\mathrm{CLBE}^{\mathrm{FO}}$ is directly given by the corresponding orbital energy which simply follows from the Koopman's theorem and FS effects correspond to the relaxation energy $\left(E_{R}\right)$ of the electron density in response to the core hole. In this case, the difference to experiment corresponds to the difference in electron correlation energy between the neutral and ionized molecule and the missing relativistic effects. In the case of DFT predicted CLBEs, the Kohn-Sham orbital energy does not provide the CLBE ${ }^{\mathrm{FO}}$ and FS effects account not only for electron density relaxation but also for the corresponding electron correlation difference although in an uncontrolled way. In the framework of DFT, the $\mathrm{CLBE}^{\mathrm{FO}}$ can also be rigorously computed as shown recently. ${ }^{13}$ The definition of initial and final state effects above applies equally to the different contributions to the $\triangle \mathrm{CLBEs}$ and thus

$$
\begin{aligned}
& \Delta \mathrm{IS}=\mathrm{CLBE}^{\mathrm{FO}}-\mathrm{CLBE}^{\mathrm{FO}} \text { (reference) } \\
& \Delta \mathrm{E}_{\mathrm{R}}=\mathrm{E}_{\mathrm{R}}-\mathrm{E}_{\mathrm{R}}(\text { reference) } \\
& \Delta \mathrm{CLBE}=\mathrm{CLBE}-\mathrm{CLBE} \text { (reference) }
\end{aligned}
$$


There are many examples showing that, for a given atomic core, $\triangle \mathrm{CLBEs}$, that include the screening of the core-hole by the overall electron density, are rather atomic in character and thus quite constant. Consequently, it is often assumed that $\triangle$ CLBEs are dominated by IS effects. ${ }^{14}$ Paradigmatic examples for IS dominated $\triangle$ CLBEs are the case of the $2 p$ level of the metal atom (M) in the bulk oxides $\mathrm{MgO}$ to $\mathrm{BaO}$ ionic crystals series relative to the gas phase $\mathrm{M}^{2+}$ cation, ${ }^{15,16}$ the $\mathrm{O}(1 \mathrm{~s})$ core level for $\mathrm{O}$ above and below a cluster model representation of the $\mathrm{Al}(111)$ surface,${ }^{17}$ the $\mathrm{N}(1 \mathrm{~s})$ core level in pyridine and pyrrole ${ }^{18}$ which indeed is due to difference in the occupancy of the $\mathrm{sp}^{3}$ hybrid orbitals in the two molecules or the ${ }^{1} \Pi-{ }^{3} \Pi$ multiplet splitting of the $N(1 \mathrm{~s})$ and $O(1 \mathrm{~s})$ CLBEs in the ${ }^{2} \Pi$ open-shell ground state of the NO molecule. ${ }^{19}$ However, one can also argue that the screening of the core hole will become progressively more important as the electron density around the core to be ionized increases. This may involve either increasing the atomic number of the atom where the core hole will be created or, for a fixed atom, increasing the atomic number of the ligands. The case of the $2 \mathrm{p}$ core level of metal atoms $\mathrm{M}$ in the $\mathrm{MgO}$ to $\mathrm{BaO}$ bulk oxides series relative to $\mathrm{M}^{2+}$, which is clearly dominated by IS effects, ${ }^{15,16}$ seems to provide a counter example to the above argument. However, a detailed analysis of $\triangle$ CLBEs in these highly ionic oxides evidences that the largest contribution arises from electrostatic effects, i.e. from the Madelung field at each site of these ionic crystals. ${ }^{15,16}$ Thus additional information is needed for heavier elements containing molecular systems where CLBEs with respect to vacuum are well-defined and where experimental information allows for a direct comparison to experiment.

In the present work we systematically investigated the trends in CLBEs for a series of molecules with the generic $\mathrm{AB}_{2}$ formula with $\mathrm{A}$ and $\mathrm{B}$ chosen from the group 14 and 16, respectively. The obtained results show that, quite surprisingly, the importance of IS effects for $\triangle$ CLBEs are strongly affected by electron correlation to the point that values predicted from Hartree-Fock calculations are severely biased even if final CLBEs are reasonably close to available experimental results or to those obtained from more accurate methods. The physical reasons behind this finding are discussed in detail and the implications of this finding for the use of Hartree-Fock in the interpretation of XPS in surface and materials science is highlighted.

\section{Model systems and computational details}

The present study of the importance of IS effects in $\triangle$ CLBEs involves a series of 9 molecules with the generic $\mathrm{AB}_{2}$ formula with $\mathrm{A}=\mathrm{C}, \mathrm{Si}$ and $\mathrm{Ge}$ and $\mathrm{B}=\mathrm{O}, \mathrm{S}, \mathrm{Se}$. These molecules contain two double bonds, which may imply a noticeable contribution of non-dynamical correlation. ${ }^{20}$ Moreover, one can investigate trends by fixing $\mathrm{A}$ and varying $\mathrm{B}$ as in the $\mathrm{CO}_{2}, \mathrm{CS}_{2}$ and $\mathrm{CSe}_{2}$ series or fix $\mathrm{B}$ and vary $\mathrm{A}$ as in going from $\mathrm{CO}_{2}$ to $\mathrm{SiO}_{2}$ and $\mathrm{GeO}_{2}$. In the first case, we analyse the $\mathrm{C}(1 \mathrm{~s})$ level whereas in the second one we analyse $\mathrm{C}(1 \mathrm{~s}), \mathrm{Si}(1 \mathrm{~s})$ and $\mathrm{Si}(2 \mathrm{~s})$ as well as $\mathrm{Ge}(1 \mathrm{~s})$, Ge (2s) and $\mathrm{Ge}(3 \mathrm{~s})$. In this way one can inspect energy levels which are too deep to be reached by experiments and, at the same time, allows 
one to see whether the trends for different core levels are the same or differ from one to another. Besides, experimental values are available for the $\mathrm{C}(1 \mathrm{~s})$ level of $\mathrm{CO}_{2}$ and $\mathrm{CS}_{2}$ which permits to assess the accuracy of the overall computational framework described below.

The molecular structure of the 9 molecules in their closed-shell, singlet, ground state has been optimized using the well-known hybrid density functional theory B3LYP method, which is known to provide a quite accurate description of a broad family of organic molecules ${ }^{21}$ and transition metal complexes, ${ }^{22}$ using the basis sets described below. The CLBEs of interest have been computed at the B3LYP geometry at various levels of theory using Eq.(1). These include the self-consistent field HartreeFock (HF), the complete active space self-consistent field $(\mathrm{CASSCF})^{23}$ and the revTPSS ${ }^{12}$ density functional theory (DFT) based method. In all cases, different technical options where used to ensure that the SCF procedure converges to the core hole (doublet) state of interest rather than a valence level. ${ }^{24}$ In the case of HF and DFT calculations this is achieved by using overlap instead of Aufbau criteria to select the occupied orbitals during the SCF procedure as is done, for instance, in the GAMESS-06 code ${ }^{25,26}$ used in the present study. In the CASSCF calculations to converge to the hole state requires several pairs of steps of fixing, or freezing, a subset of the orbital space while the remaining orbitals were varied as described in detail by Pueyo-Bellafont et al. ${ }^{27}$ The CASSCF calculations have been carried out using the MOLCAS ${ }^{28}$ code.

The reason to include CASSCF and revTPSS is to allow for non-dynamical correlation effects arising from an incorrect balance of the many valence bond forms in the HF wave function. ${ }^{20}$ Precisely, recent work has shown that these effects can make an important contribution to the final CLBEs. ${ }^{27} \mathrm{We}$ will show that this is also the case for the molecules studied in the present work. For a valence active space, the multireference CASSCF approach rigorously accounts for non-dynamic or molecular electron correlation effects $^{20,23}$ coming from near-degeneracies of various electronic states. These effects can be different for the initial neutral and the final core hole ionized states and therefore, affect the binding energy. On the other hand, revTPSS has been found to be one of the few DFT based approaches that are able to mimic CASSCF results for CLBEs. Moreover, for the 185 CLBEs dataset described above, revTPSS has been found to be the DFT method with smallest mean absolute error. ${ }^{10}$ Notice, however, that rev-TPSS accounts for all electron correlation effects through the exchange-correlation functional. In this case no distinction can be made between non-dynamic and dynamic (mainly atomic in nature) electron correlation effects as both are, in principle, included, even if to an undetermined extent. The CLBEs computed applying Eq. (1) include both, initial and final state effects. To separate initial and final state contributions we make use of Eq. (2). For technical reasons, this is carried out at the HF and TPSS levels.

The total HF energies needed to compute the CLBEs as in Eq. (1) and (2) have been obtained using three different Gaussian Type Orbitals (GTO) basis sets hereafter referred to as Basis 1, Basis 2 and Basis 3. Basis 1 starts from the primitive GTO sets used to build the aug-cc-pVQZ family for C and O, ${ }^{29}$ 
$\mathrm{Si}$ and $\mathrm{S},{ }^{30}$ and $\mathrm{Ge}$ and $\mathrm{Se}^{31}$ with exponents taken from the EMSL site ${ }^{32}$ and fully uncontracted it, a necessary step to allow for sufficient flexibility in describing the final, core ionized, state. Hence the Basis 1 for $\mathrm{C}$ and $\mathrm{O}$ can be denoted as (13s, 8p, 4d, 3f, 2g), that of Si and $\mathrm{S}$ as (17s, 12p, 4d, 3f, 2g) and, finally, that of Ge and Se as $(22 \mathrm{~s}, 17 \mathrm{p}, 13 \mathrm{~d}, 3 \mathrm{f}, 2 \mathrm{~g})$. For the properties of interest in the present work it is clear that $\mathrm{f}$ and $\mathrm{g}$ primitives are not really needed and are included just for consistency. Nevertheless, this is further checked by Basis 2, which differs from Basis 1 just on the $\mathrm{f}$ and $\mathrm{g}$ functions which are removed. Accordingly, Basis 2 for $\mathrm{C}$ and $\mathrm{O}$ is $(13 \mathrm{~s}, 8 \mathrm{p}, 4 \mathrm{~d})$, that of $\mathrm{Si}$ and $\mathrm{S}(17 \mathrm{~s}, 12 \mathrm{p}, 4 \mathrm{~d})$ and that of Ge and $\mathrm{Se}$ $(22 \mathrm{~s}, 17 \mathrm{p}, 13 \mathrm{~d})$. Basis 3 is obtained from Ahlrichs valence triple- $\zeta$ plus polarization basis ${ }^{33}$, again with exponents taken from the EMSL site ${ }^{32}$ and fully uncontracted becoming (10s, 6p, 1d) for C and O, (12s, $9 \mathrm{p}, 1 \mathrm{~d})$ for Si and $\mathrm{S}$ and $(14 \mathrm{~s}, 11 \mathrm{p}, 6 \mathrm{~d})$ for Ge and Se. For convenience, details of these basis sets are given in the Electronic Supplementary Information (ESI). In the forthcoming section we will show that the HF results obtained with the three basis sets are almost identical. For simplicity, CASSCF and revTPSS calculations presented here correspond to Basis 3. In general, DFT based methods do not strongly depend on the basis set. For the CASSCF calculations we checked the effect of the basis size on the CLBEs by computing the $\mathrm{C}(1 \mathrm{~s})$ binding energy for $\mathrm{CO} 2$ and $\mathrm{CS} 2$ applying the three previously commented basis sets. For both molecules, going from the largest Basis 1 to Basis 2 the CLBEs increase by $0.1 \mathrm{eV}$ only, and decreasing further the basis set size from Basis 2 to Basis 3 makes the CLBEs nearly $0.1 \mathrm{eV}$ larger. For completeness, values from the original $\operatorname{TPSS}^{34}$ functional have also been obtained and are reported in the supporting information. The CASSCF calculations have been carried out considering 8 electrons and the 6 orbitals of $\pi$ character defining the $A=B$ double bonds. Following the standard notation, the final active space is referred to as $\operatorname{CAS}(8,6)$.

To conclude this section, we note that relativistic effects make a small but noticeable contribution to the calculated CLBEs. Nevertheless, this contribution is essentially atomic in character. This is confirmed by results for the $\mathrm{CO}$ molecule obtained by means of four-component Dirac-HartreeFock calculations where relativistic contributions to CLBEs are nearly identical to those for the isolated C and $\mathrm{O}$ atoms using the same approach. ${ }^{35}$ These corrections increase the calculated non-relativistic CLBE by $0.13 \mathrm{eV}$ for $\mathrm{C}(1 \mathrm{~s})$ and $0.45 \mathrm{eV}$ for $\mathrm{O}(1 \mathrm{~s})$.

\section{Results and discussion}

To facilitate reproducibility of the present results, Table 1 reports the A-B distances as predicted from calculations using the B3LYP method and each one of the three basis sets commented above. Note also that the 9 molecules exhibit a linear molecular structure, the calculated vibrational frequencies reported confirm that all correspond to minima in the potential energy surface (see Table S1 in the ESI). Inspection of Table 1 also shows that, as far as interatomic distances are concerned, the three basis sets provide nearly the same results with deviations of at most $0.01 \AA$. Overall, the A-B distances increase when going down in a given group. Changing $\mathrm{C}$ by Si increases the interatomic distances by $0.35 \AA$, 
while replacing Si for Ge the enlargement is only $0.1 \AA$. Similarly, changing the atom of group 16 from O to $\mathrm{S}$ an increase of the distance of $0.4 \AA$ is observed while substituting $\mathrm{S}$ for Se the distance increases $0.14 \AA ̊$.

\section{Initial and Final State Hartree-Fock CLBES}

We start the discussion by focussing on the 1 s core level of C, Si and Ge along the three series of molecules. Table 2 summarizes the set of results for the CLBEs as predicted from the Koopman's theorem (Eq. 2) and from total energy differences $(\triangle \mathrm{SCF})$ as in Eq. 1. The total energy values for the ground and for the core ionized states are reported in Table S2 of the ESI. Table 2 also reports the contribution of the final state effects to the CLBEs, which are labelled as $E_{R}$ since they are dominated by the relaxation of the electron density in response to the presence of the core hole.

The analysis of Table 2 reveals many interesting features. First, the effect of the basis set on the calculates IS and $\triangle \mathrm{SCF}$ CLBEs is rather small seldom, of the order of $0.1 \mathrm{eV}$ for the C(1s) CLBEs and reaching at most $0.5 \mathrm{eV}$ for the $\mathrm{Ge}(1 \mathrm{~s})$. Note, however, that the absolute values for $\mathrm{Si}(1 \mathrm{~s})$ and $\mathrm{Ge}(1 \mathrm{~s})$ is larger than $10000 \mathrm{eV}$ which makes the relative effect of the basis set nearly negligible indicating that the basis sets used are extended enough. The second interesting feature concerns the magnitude of $E_{R}$ which although much smaller than the final CLBEs makes a significant contribution, approaching the calculated values to those measured in XPS experiments. ${ }^{6,7,910}$ Not surprisingly, $E_{R}$ increases from $C$ to Ge which is simply due to the increase in electron density with increasing atomic number. Nevertheless, the most salient feature of this set of calculations is the unexpected rather large variation of $E_{R}$ for the core hole of the central atom (A) as the atomic number of the atoms bonded to it (B) increases. This would have consequences for the analysis of the $\triangle$ CLBEs along a given series since, as shown in Table 3 , are no longer dominated by initial state effects, which is against evidence in many systems. ${ }^{6,14}$ Here, it is important to point out that $\triangle \mathrm{CLBEs}$ imply choosing a reference which may be arbitrary. The choice is different depending on the purpose of the analysis. For instance, in a previous work related to the performance of DFT methods in describing trends in CLBEs, the $\mathrm{N}(1 \mathrm{~s})$ in $\mathrm{NH}_{3}$ was taken as reference. ${ }^{13}$ This choice allowed to show that, even if Kohn-Sham orbital energies do not provide a measure of initial state effects, their shift with respect to a reference closely followed the rigorously theoretically grounded Hartree-Fock initial state trends from orbital energies with obvious consequences for interpretation of XPS data from DFT based calculations. ${ }^{36}$ Of course, such a choice is not aimed at providing differences in chemical bonding along the series of N-containing molecules there studied. Another completely different situation is found when comparing $\triangle$ CLBEs in similar molecules as for instance $\mathrm{N}(1 \mathrm{~s})$ in pyridine and pyrrole where the separation into initial and final states clearly shows that the difference is dominated by initial state effects which, in turn, are related to the different occupation of the hybrid $\mathrm{sp}^{2}$ 
orbitals. ${ }^{18}$ In this sense, the comparison of $\triangle \mathrm{CLBEs}$ along the $\mathrm{AB}_{2}$ series, as described in detail below, is physically meaningful.

Table 3 reports the HF calculated $\Delta \mathrm{IS}, \Delta \mathrm{E}_{\mathrm{R}}$ and $\triangle \mathrm{SCF}$ values for $\Delta \mathrm{CLBEs}$ corresponding to $1 \mathrm{~s}$ ionization of the central atoms $(\mathrm{C}, \mathrm{Si}, \mathrm{Ge})$ of the molecules in the series, relative to the molecules with oxygen. Inspection of Table 3 reveals some interesting features additional to those discussed regarding Table 3 in relationship to the influence of the basis set. In fact, Table 3 shows that $\triangle \mathrm{CLBEs}$ for $\mathrm{CS}_{2}$ and $\mathrm{CSe}_{2}$ relative to $\mathrm{CO}_{2}$ have contributions from both, initial and final state, effects. The final state effects dominate but initial state contributions provide a qualitatively correct trend indicating that, to a significant extent, differences in chemical bonding between $\mathrm{CO}_{2}$ and $\mathrm{CS}_{2}$ and $\mathrm{CSe}_{2}$ are already present in the neutral molecule and, hence, XPS measured CLBEs and their shifts provides difference in chemical bond along this series. However, a completely different situation appears when analysing the equivalent molecules with Si or Ge where the contribution of initial effects is negligible and may even be qualitatively wrong. The corollary from this quite surprising and unexpected finding would be that XPS measured CLBEs and their shifts do not provide information regarding differences in chemical bonding in the neutral molecules since the overall effect arises from the core-hole ionized state. However, such a strong statement needs further analysis. In fact, results in Table 3 seem to indicate that the core-hole is fully screened by the increasing electron density around the core region. Since these results correspond to the $1 \mathrm{~s}$ ionization in Si and Ge containing molecules, one may argue that other core levels will exhibit a less marked screening of the core hole. To investigate this possibility, calculations have been carried out for the ionization from the $2 \mathrm{~s}$ level in the Si containing molecules and from the $2 \mathrm{~s}$ and $3 \mathrm{~s}$ in the Ge containing ones. In the view of the small influence of the employed basis set, these additional calculations have been carried out using Basis 3 only. Results for the Si (2s), Ge(2s) and Ge(3s) CLBEs and the corresponding shifts with respect to either $\mathrm{SiO}_{2}$ or $\mathrm{GeO}_{2}$ are reported in Tables 4 and 5 respectively whereas the total energy values obtained in the HF calculations are reported in Table S3 of the ESI. The trends for these more external core levels are as for the $1 \mathrm{~s}$ discussed previously and dominated by final state effects.

The results presented so far indicate that increasing the atomic number of the atoms where the core hole is produced or that of the atoms bonded to it may result in a complete screening of the core hole. This would imply a complete loss of information from initial state effects, which may have consequences in the use of XPS to analyse the chemical bonding in situations implying these atoms. At this point, one may argue that the overall discussion relies in results obtained by means of HF calculations which, even often leading to a good estimate of the measured CLBEs, may not be accurate enough to describe the subtleties of the CLBEs for the systems of interest in the present work. To check whether this is the case, next section describes results from CASSCF and revTPSS calculations, which account for the important electron correlation effects and provide also comparison to available experimental results. 
Experimental results are available for the $\mathrm{C}(1 \mathrm{~s})$ level of gas phase $\mathrm{CO}_{2}$ and $\mathrm{CS}_{2}$ only, the corresponding values being $297.6 \mathrm{eV}$ and $293.1 \mathrm{eV}$, respectively. These compare very well with values reported in Table 2 for any of the three basis employed; i.e. 299.5 and $295.0 \mathrm{eV}$ for Basis 3. Moreover, the agreement is excellent for the $\triangle$ CLBEs between these two molecules. However, there is a detail that shows that, in spite of the numerical agreement, something is somehow wrong. In fact, the calculated HF $(\triangle \mathrm{SCF})$ values are larger than experiment whereas from the fact that electron correlation effects will be larger for the neutral molecule than for the cation, one would expect the opposite. In fact, recent work has shown that this is precisely the fingerprint that important electron correlation effects are missing. Calculated results at the CASSCF level using the CAS(8,6) and Basis 3 predict values of 297.4 and $293.2 \mathrm{eV}$, the first below the experimental result as expected and the second slightly above, indicating that some nondynamical correlation has been left out. To be rigorous, one would need to add the $0.13 \mathrm{eV}$ relativistic contribution to the $\mathrm{C}(1 \mathrm{~s})$ binding energy calculated from a non-relativistic method so that the $\mathrm{C}(1 \mathrm{~s})$ CLBE for $\mathrm{CO}_{2}$ becomes in perfect agreement with experiment whereas the corresponding value for $\mathrm{CS}_{2}$ is slightly overestimated. The preceding discussion shows that non-dynamical correlation makes a modest contribution to the CLBEs of the molecules here studied decreasing the calculated HF CLBEs by 0.8-2.1 $\mathrm{eV}$ (Table 6.). It is worth noting that the contribution of non-dynamical electron correlation to the CLBEs is larger for the $\mathrm{C}(1 \mathrm{~s})$ core hole in the three $\mathrm{C}$-containing molecules than for the $\mathrm{Si}(1 \mathrm{~s})$ and $\mathrm{Ge}(1 \mathrm{~s})$ CLBEs of the remaining molecules. This can be explained by the proximity of the valence $\pi$ orbitals included in the active space to the $\mathrm{C}(1 \mathrm{~s})$ core hole, the interaction becoming less important for the deeper $1 \mathrm{~s}$ core levels corresponding to Si and Ge molecules. The full set of total energy values for the neutral and core ionized molecules is reported in Table S4 of the ESI. To further illustrate the importance of non-dynamic correlation effects, one can rely in the weight of the different configurations to the CASSCF wave function or, alternatively, in the natural occupation numbers of the active orbitals. For instance, for the $\mathrm{CO}_{2}$ molecule, the main configuration in the ground state wave function has a weight of 0.94 while for the hole state the largest weight is 0.87 . These values change to 0.96 and 0.91 for $\mathrm{SiO}_{2}$ (similar values are found for $\mathrm{GeO}_{2}$ ). This confirms that non-dynamical correlation effects are more important for the $\mathrm{C}(1 \mathrm{~s})$ core hole that for $\mathrm{Si}(1 \mathrm{~s})$ and $\mathrm{Ge}(1 \mathrm{~s})$, as shown in Table 6. For $\mathrm{CO}_{2}$ we have even considered larger active spaces, in particular a CAS containing 8 orbitals ( $\pi$ and $\sigma$ orbitals) and 10 electrons. Result for the $C(1 \mathrm{~s})$ CLBE was $0.17 \mathrm{eV}$ smaller than the one obtained by the CAS(8,6), $297.21 \mathrm{eV}$ versus $297.39 \mathrm{eV}$. Inspection to the wave functions showed that the relevant molecular orbitals are already included in the CAS $(8,6)$ calculations.

The contribution of non-dynamic electron correlation to the final CLBEs is certainly small but it is of the order the $\triangle \mathrm{CLBEs}$ of the $\mathrm{S}$ and Se containing molecules relative to the ones with $\mathrm{O}$ as shown in Tables 3 or 5 at the HF level. Therefore, one may argue that electron correlation also affects the decomposition into initial and final state contributions. To analyse the influence of electron correlation in 
that decomposition, we rely on the revTPSS density functional that has shown to provide a physically meaningful performance in the case of $\mathrm{CO} .^{27} \mathrm{~A}$ similar good performance is found in the present work for the CBLEs for the $1 \mathrm{~s}$ core levels of the set of molecules under study. The $\triangle \mathrm{SCF}$ column in Table 7 reports the CLBEs as predicted from this DFT based method, the difference with respect to the CASSCF values in Table 6 is also included below the $\triangle \mathrm{CAS}$ entry. The agreement between revTPSS and CASSCF calculated CLBEs is really remarkable indicating that this method is able to capture the non-dynamic correlation effects, as shown earlier for the case of $\mathrm{CO} .{ }^{27}$ Note how the revTPSS C(1s) CLBE for $\mathrm{CO}_{2}$ and $\mathrm{CS}_{2}$ is very close to the experimental value and also slightly smaller than it. The fact that revTPSS provides a description so close to that of the CASSCF method allows us to investigate the decomposition into initial and final states because the FO values can be obtained in a rigorous and straightforward way. ${ }^{13}$ A FO estimate at the CASSCF level is also possible as shown in the case of the $\mathrm{CO}^{27}$ although technically is more involved and will be difficult to reproduce by others as it requires non trivial manipulation of the corresponding code. The decomposition of revTPSS CLBEs into initial and final state contributions is included in Table 7. The revTPSS total energy for the neutral molecule, the frozen orbital and variational energy of the core ionized molecules is reported in Table S5 of the ESI while the equivalent information for TPSS is reported in Table S6. Results from revTPSS and TPSS are very similar and the discussion is hence based on the revTPSS ones. Inspection of Table 7 shows that the absolute values of CLBEs as well as IS and $E_{R}$ contributions are very similar to those reported in Table 2 obtained from the HF method. This is not surprising since the differences are very subtle starting from the fact that, as mentioned, HF CLBEs for the $\mathrm{C}(1 \mathrm{~s})$ level in $\mathrm{CO}_{2}$ and $\mathrm{CS}_{2}$ are larger than experiment whereas revTPSS values are as expected smaller than experiment and also close to those predicted by the CASSCF method. Note the difference between revTPSS and CASSCF final CLBEs values is small for the C(1s) levels inspected, slightly larger for the $\mathrm{Si}(1 \mathrm{~s})$ ones and even larger for those of $\mathrm{Ge}(1 \mathrm{~s})$. This is to be expected since CASSCF only captures a fraction of the electron correlation, in principle, most of the non-dynamic correlation effects, whereas revTPSS is supposed to account for all electron correlation energy. For the 1s core hole in the $\mathrm{C}$ containing molecules, the major contribution to the electron correlation is already included in the active space, dynamic correlation being much less important. RevTPSS satisfactorily captures these effects as can be inferred from Table 7. However, when the 1s hole state is created in third and four row atoms, dynamic correlation, which is not included in the CASSCF calculations, becomes more important. This explains the difference between CASSCF and revTPSS calculations and allows to qualitatively evaluate the relative importance of dynamical electron correlation, being six times larger for $\mathrm{Si}(1 \mathrm{~s})$ compared to $\mathrm{C}(1 \mathrm{~s})$ and, likewise, for $\mathrm{Ge}(1 \mathrm{~s})$ the contribution is six time larger than for $\mathrm{Si}(1 \mathrm{~s})$.

Finally, we comment on the $\triangle$ CLBEs of the $\mathrm{C}(1 \mathrm{~s}), \mathrm{Si}(1 \mathrm{~s})$ and $\mathrm{Ge}(1 \mathrm{~s})$ levels relative to the systems containing oxygen with values summarized in Table 8. Contrarily, to what is found at the HF level (Table 3 ), the decomposition of $\triangle$ CLBEs into initial and final states shows a more balanced picture 
where, even if final state contributions are important, the main trends are already provided by initial effects contributions. This is in agreement with arguments and results provided in previous works ${ }^{6,14}$ and supports the interpretation of $\triangle$ CLBEs measured by XPS as indicative of differences in the chemical bonding of the neutral, unionized systems. Note also that, in cases where non-dynamic electron correlation appears to be noticeable, the decomposition at the HF level may lead to artefacts and erroneous conclusions.

\section{Conclusions}

Systems with atoms in different chemical environment exhibit differences in their core level shift binding energies, which are accurately measured by X-ray photoemission spectroscopy. These binding energies can be quite accurately reproduced by theoretical methods of electronic structure and, more importantly, the decomposition of the core level binding energies and their shifts permits to quantify to which extent the measured shift can be attributed to the differences in the systems object of study or if, on the contrary, they are providing information of the core hole containing systems. The present study has been carried out for a series of molecules where, due to the presence of cumulated double bonds, nondynamic correlation is important and the combination of increasing number of electrons on the atoms to be ionized and/or those bonded to it and the effect of electron delocalization may suggest that a complete screening of the core-hole takes place. In this way, the systems chosen constitute one of the worst possible scenarios for such decomposition.

The series of results obtained by means of Hartree-Fock, CASSCF and the revTPSS methods show that, whereas these methods provide quite accurate estimates of absolute values of core level binding energies, a meaningful decomposition into initial and final states requires accounting for electron correlation effects, the Hartree-Fock decomposition being physically incorrect. The use of DFT based methods can provide a physically meaningful decomposition but, since not all functionals are able to incorporate the non-dynamic correlation effects in a balanced way, ${ }^{27}$ it is necessary to make use of CASSCF or equivalent wave function based methods to properly validate the choice of a given DFT based method. Since DFT based methods are broadly used to study the atomic and electronic structure of solids, surfaces and adsorbates thereon, it is necessary to take the interpretations based on inspection of Kohn-Sham orbital energies, aimed at providing a measure of initial state effect contribution, ${ }^{36}$ with special caution.

\section{Conflicts of interest}

There are no conflicts to declare

\section{Acknowledgements}

The authors wish to acknowledge the continued discussion with Dr. Paul S. Bagus and his many interesting and pertinent remarks. This research was supported by the Spanish MINECO/FEDER 
CTQ2015-64618-R and MICINN Spanish Structures of Excellence María de Maeztu program through grant MDM-2017-0767 and, in part, by Generalitat de Catalunya (grants 2017SGR13 and XRQTC). F. I. acknowledges additional support from the 2015 ICREA Academia Award for Excellence in University Research. 
Table 1. Internuclear distances for the set of molecules studied in the present work as predicted by the B3LYP method and the three different GTO basis sets (see text). All results are in $\AA$.

\begin{tabular}{lccc}
\hline & C series & Si series & Ge series \\
\hline & $\mathrm{d}(\mathrm{C}-\mathrm{O})$ & $\mathrm{d}(\mathrm{Si}-\mathrm{O})$ & $\mathrm{d}(\mathrm{Ge}-\mathrm{O})$ \\
\hline Basis 1 & 1.159 & 1.512 & 1.622 \\
\hline Basis 2 & 1.160 & 1.513 & 1.626 \\
\hline Basis 3 & 1.161 & 1.525 & 1.629 \\
\hline & $\mathrm{d}(\mathrm{C}-\mathrm{S})$ & $\mathrm{d}(\mathrm{Si}-\mathrm{S})$ & $\mathrm{d}(\mathrm{Ge}-\mathrm{S})$ \\
\hline Basis 1 & 1.555 & 1.930 & 2.012 \\
\hline Basis 2 & 1.557 & 1.933 & 2.018 \\
\hline Basis 3 & 1.563 & 1.941 & 2.019 \\
\hline Basis 1 & $\mathrm{d}(\mathrm{C}-\mathrm{Se})$ & $\mathrm{d}(\mathrm{Si}-\mathrm{Se})$ & $\mathrm{d}(\mathrm{Ge}-\mathrm{Se})$ \\
\hline Basis 2 & 1.697 & 2.067 & 2.145 \\
\hline Basis 3 & 1.701 & 2.072 & 2.151 \\
\hline
\end{tabular}


Table 2. Core level binding energies for the set of molecules studied in the present work as predicted by the HF method using the geometries reported in Table 1 and the three different GTO basis sets. IS stands for the initial state computed CLBES whereas $\triangle \mathrm{SCF}$ includes initial and final states. The contribution of final state effects is the difference between IS and $\triangle \mathrm{SCF}$ here denoted as $\mathrm{E}_{\mathrm{R}}$ and reported in the rightmost column. All results correspond to ionization of the 1 s level and are in $\mathrm{eV}$.

\begin{tabular}{|c|c|c|c|c|c|}
\hline Core & Molecule & Basis & IS & $\triangle \mathrm{SCF}$ & $\mathbf{E}_{\mathbf{R}}$ \\
\hline \multirow[t]{9}{*}{$\mathrm{C}(1 \mathrm{~s})$} & $\mathrm{CO}_{2}$ & 1 & 312.0 & 299.5 & -12.5 \\
\hline & & 2 & 311.9 & 299.4 & -12.5 \\
\hline & & 3 & 312.0 & 299.5 & -12.5 \\
\hline & $\mathrm{CS}_{2}$ & 1 & 310.5 & 295.0 & -15.5 \\
\hline & & 2 & 310.4 & 294.9 & -15.5 \\
\hline & & 3 & 310.5 & 295.0 & -15.5 \\
\hline & $\mathrm{CSe}_{2}$ & 1 & 310.4 & 294.3 & -16.2 \\
\hline & & 2 & 310.5 & 294.3 & -16.2 \\
\hline & & 3 & 310.4 & 294.3 & -16.2 \\
\hline \multirow[t]{9}{*}{$\operatorname{Si}(1 s)$} & $\mathrm{SiO}_{2}$ & 1 & 1874.7 & 1848.4 & -26.4 \\
\hline & & 2 & 1874.4 & 1847.9 & -26.5 \\
\hline & & 3 & 1874.7 & 1848.4 & -26.4 \\
\hline & $\mathrm{SiS}_{2}$ & 1 & 1874.9 & 1846.6 & -28.2 \\
\hline & & 2 & 1874.9 & 1846.4 & -28.3 \\
\hline & & 3 & 1874.9 & 1846.6 & -28.2 \\
\hline & $\mathrm{SiSe}_{2}$ & 1 & 1874.8 & 1846.2 & -28.6 \\
\hline & & 2 & 1874.7 & 1846.1 & -28.8 \\
\hline & & 3 & 1874.8 & 1846.2 & -28.6 \\
\hline \multirow[t]{9}{*}{$\mathrm{Ge}(1 \mathrm{~s})$} & $\mathrm{GeO}_{2}$ & 1 & 11030.3 & 10980.4 & -49.9 \\
\hline & & 2 & 11029.8 & 10979.8 & -50.1 \\
\hline & & 3 & 11030.3 & 10980.4 & -49.9 \\
\hline & $\mathrm{GeS}_{2}$ & 1 & 11030.2 & 10978.9 & -51.3 \\
\hline & & 2 & 11029.8 & 10978.4 & -51.4 \\
\hline & & 3 & 11030.2 & 10978.9 & -51.3 \\
\hline & $\mathrm{GeSe}_{2}$ & 1 & 11030.1 & 10978.5 & -51.5 \\
\hline & & 2 & 11029.9 & 10978.1 & -51.8 \\
\hline & & 3 & 11030.1 & 10978.5 & -51.5 \\
\hline
\end{tabular}


Table 3. Core level binding energies shifts ( $\triangle$ CLBEs) for the set of molecules studied in the present work as predicted by the HF method using the geometries reported in Table 1 and the three different GTO basis sets. $\Delta \mathrm{IS}$ stands for the initial state computed shifts (Eq. 3), $\Delta \mathrm{E}_{\mathrm{R}}$ corresponds to the difference in final state contributions (Eq.4) and $\triangle$ CLBEs (Eq. 5) includes initial and final states. For each of the central atoms $(\mathrm{C}, \mathrm{Si}, \mathrm{Ge})$, the $\triangle \mathrm{CLBEs}$ are relative to the molecule with oxygen. All results correspond to ionization of the $1 \mathrm{~s}$ level and are in $\mathrm{eV}$.

\begin{tabular}{cccccc}
\hline Reference & Molecule & Basis & $\Delta \mathbf{I S}$ & $\Delta \mathbf{E}_{\mathbf{R}}$ & $\Delta$ CLBEs \\
\hline $\mathrm{C}(1 \mathrm{~s})$ in $\mathrm{CO}_{2}$ & $\mathrm{CS}_{2}$ & 1 & -1.5 & -3.0 & -4.5 \\
\hline & & 2 & -1.5 & -3.0 & -4.5 \\
\hline & & 3 & -1.5 & -3.0 & -4.5 \\
\hline & $\mathrm{CSe}_{2}$ & 1 & -1.6 & -3.6 & -5.2 \\
\hline & & 2 & -1.4 & -3.7 & -5.1 \\
\hline $\mathrm{Si}(1 \mathrm{~s}){\text { in } \mathrm{SiO}_{2}}$ & & 3 & -1.6 & -3.6 & -5.2 \\
\hline & $\mathrm{SiS}_{2}$ & 1 & 0.2 & -1.8 & -1.6 \\
\hline & & 2 & 0.5 & -1.5 & -1.0 \\
\hline & & 3 & 0.2 & -1.8 & -1.6 \\
\hline & & 1 & 0.1 & -2.2 & -2.1 \\
\hline $\mathrm{SiSe}$ & & 0.3 & -1.8 & -1.5 \\
\hline & & 3 & 0.1 & -2.2 & -2.1 \\
\hline & & 1 & -0.1 & -1.5 & -1.6 \\
\hline & & 2 & 0.0 & -1.4 & -1.4 \\
\hline & & 3 & -0.1 & -1.5 & -1.6 \\
\hline & & 1 & -0.2 & -1.9 & -2.1 \\
\hline & & 2 & 0.1 & -1.7 & -1.6 \\
\hline
\end{tabular}


Table 4. Core level binding energies for the $2 \mathrm{~s}$ level of the $\mathrm{Si}$ and $2 \mathrm{~s}$ and $3 \mathrm{~s}$ levels of the Ge containing molecules studied in the present work as predicted by the HF method using the geometries reported in Table 1 and Basis 3. IS, $\Delta \mathrm{SCF}$ and $\mathrm{E}_{\mathrm{R}}$ are as in Table 2. All results are in $\mathrm{eV}$.

\begin{tabular}{ccccc}
\hline Core & Molecule & IS & $\Delta \mathbf{S C F}$ & $\mathbf{E}_{\mathbf{R}}$ \\
\hline $\mathrm{Si}(2 \mathrm{~s})$ & $\mathrm{SiO}_{2}$ & 169.9 & 163.5 & -6.4 \\
\hline & $\mathrm{SiS}_{2}$ & 169.9 & 162.0 & -8.0 \\
\hline & $\mathrm{SiSe}_{2}$ & 169.9 & 161.6 & -8.3 \\
\hline $\mathrm{Ge}(2 \mathrm{~s})$ & $\mathrm{GeO}_{2}$ & 1421.9 & 1396.3 & -25.7 \\
\hline & $\mathrm{GeS}_{2}$ & 1421.9 & 1394.9 & -27.0 \\
\hline & $\mathrm{GeSe}_{2}$ & 1421.8 & 1394.5 & -27.2 \\
\hline $\mathrm{Ge}(3 \mathrm{~s})$ & $\mathrm{GeO}_{2}$ & 198.6 & 191.0 & -7.6 \\
\hline & $\mathrm{GeS}_{2}$ & 198.5 & 189.6 & -8.9 \\
\hline & $\mathrm{GeSe}_{2}$ & 198.3 & 189.2 & -9.1 \\
\hline
\end{tabular}


Table 5. Core level binding energies shifts ( $\triangle$ CLBEs) for the $2 \mathrm{~s}$ level of the subset of molecules containing Si and for the $2 \mathrm{~s}$ and $3 \mathrm{~s}$ levels of molecules containing Ge. Results correspond HF calculations using the geometries reported in Table 1 and Basis 3. $\Delta \mathrm{IS}, \Delta \mathrm{E}_{\mathrm{R}}$ and $\Delta \mathrm{SCF}$ are as in Table 3 and $\Delta \mathrm{CLBEs}$ are relative to the molecules with oxygen. All results are in $\mathrm{eV}$.

\begin{tabular}{ccccc}
\hline Reference & Molecule & $\boldsymbol{\Delta} \mathbf{I S}$ & $\boldsymbol{\Delta} \mathbf{E}_{\mathbf{R}}$ & $\boldsymbol{\Delta S C F}$ \\
\hline $\mathrm{Si}(2 \mathrm{~s})$ in $\mathrm{SiO}_{2}$ & $\mathrm{SiS}_{2}$ & 0.0 & -1.5 & -1.5 \\
\hline & $\mathrm{SiSe}_{2}$ & 0.0 & -0.4 & -0.4 \\
\hline $\mathrm{Ge}(2 \mathrm{~s})$ in $\mathrm{GeO}_{2}$ & $\mathrm{GeS}_{2}$ & 0.0 & -1.4 & -1.4 \\
\hline & $\mathrm{GeSe}_{2}$ & -0.1 & -1.8 & -1.9 \\
\hline $\mathrm{Ge}(3 \mathrm{~s})$ in $\mathrm{GeO}_{2}$ & $\mathrm{GeS}_{2}$ & -0.1 & -1.4 & -1.5 \\
\hline & $\mathrm{GeSe}_{2}$ & -0.3 & -1.8 & -2.1 \\
\hline
\end{tabular}


Table 6. Core level binding energies for the set of molecules studied in the present work as predicted by the CASSCF method using the geometries reported in Table 1 and Basis $3 . \Delta \mathrm{E}_{\text {corr }}$ correspond to the contribution of non-dynamic correlation to the CLBES, i.e. the difference with respect to $\triangle \mathrm{SCF}$ values on Table 1. All results correspond to ionization of the $1 \mathrm{~s}$ level and are in $\mathrm{eV}$.

\begin{tabular}{cccc}
\hline Core & Molecule & CASSCF & $\Delta \mathbf{E}_{\text {corr }}$ \\
\hline $\mathrm{C}(1 \mathrm{~s})$ & $\mathrm{CO}_{2}$ & 297.4 & -2.1 \\
\hline & $\mathrm{CS}_{2}$ & 293.2 & -1.8 \\
\hline & $\mathrm{CSe}_{2}$ & 292.5 & -1.8 \\
\hline $\mathrm{Si}(1 \mathrm{~s})$ & $\mathrm{SiO}_{2}$ & 1847.5 & -0.9 \\
\hline & $\mathrm{SiS}_{2}$ & 1845.6 & -1.0 \\
\hline $\mathrm{SiSe}$ & 1845.2 & -1.0 \\
\hline & $\mathrm{GeO}_{2}$ & 10979.7 & -0.7 \\
\hline & $\mathrm{GeS}_{2}$ & 10978.1 & -0.8 \\
\hline & $\mathrm{GeSe}_{2}$ & 10977.7 & -0.8 \\
\hline
\end{tabular}


Table 7. Core level binding energies for the set of molecules studied in the present work as predicted by the revTPSS method using the geometries reported in Table 1 and Basis 3 . IS, $\triangle \mathrm{SCF}$, and $\mathrm{E}_{\mathrm{R}}$ are as in Table 2 whereas $\triangle$ CAS corresponds to the difference to the CASSCF values in Table 6 . All results correspond to ionization of the $1 \mathrm{~s}$ level and are in $\mathrm{eV}$.

\begin{tabular}{cccccc}
\hline Core & Molecule & IS & $\Delta \mathbf{S C F}$ & $\mathbf{E}_{\mathbf{R}}$ & $\Delta \mathbf{C A S}$ \\
\hline $\mathrm{C}(1 \mathrm{~s})$ & $\mathrm{CO}_{2}$ & 310.7 & 297.5 & -13.1 & 0.1 \\
\hline & $\mathrm{CS}_{2}$ & 309.2 & 293.4 & -15.9 & 0.2 \\
\hline & $\mathrm{CSe}_{2}$ & 310.0 & 292.7 & -17.3 & 0.2 \\
\hline $\mathrm{Si}(1 \mathrm{~s})$ & $\mathrm{SiO}_{2}$ & 1875.0 & 1846.3 & -28.7 & -1.2 \\
\hline & $\mathrm{SiS}_{2}$ & 1874.0 & 1844.5 & -29.5 & -1.1 \\
\hline $\mathrm{Se}(1 \mathrm{~s})$ & $\mathrm{SiSe}_{2}$ & 1874.4 & 1844.0 & -30.4 & -1.2 \\
\hline & $\mathrm{GeO}_{2}$ & 11025.5 & 10972.9 & -52.7 & -6.8 \\
\hline & $\mathrm{GeS}_{2}$ & 11024.6 & 10971.4 & -53.2 & -6.7 \\
\hline & $\mathrm{GeSe}_{2}$ & 11024.9 & 10971.0 & -53.9 & -6.7 \\
\hline
\end{tabular}


Table 8. Core level binding energies shifts ( $\triangle$ CLBEs) for the set of molecules studied in the present work as predicted by the revTPSS method using the geometries reported in Table 1 and Basis $3 . \Delta \mathrm{IS}, \Delta \mathrm{E}_{\mathrm{R}}$ and $\triangle$ CLBEs are as in Table 3. For each of the central atoms $(\mathrm{C}, \mathrm{Si}, \mathrm{Ge})$, the $\triangle \mathrm{CLBEs}$ are relative to the molecule with oxygen. All results correspond to ionization of the 1s level and are in $\mathrm{eV}$.

\begin{tabular}{ccccc}
\hline Reference & Molecule & $\boldsymbol{\Delta} \mathbf{I S}$ & $\boldsymbol{\Delta} \mathbf{E}_{\mathbf{R}}$ & $\Delta$ CLBEs \\
\hline $\mathrm{C}(1 \mathrm{~s})$ in $\mathrm{CO}_{2}$ & $\mathrm{CS}_{2}$ & -1.5 & -2.7 & -4.2 \\
\hline & $\mathrm{CSe}_{2}$ & -0.7 & -4.2 & -4.9 \\
\hline $\mathrm{Si}(1 \mathrm{~s})$ in $\mathrm{SiO}_{2}$ & $\mathrm{SiS}_{2}$ & -1.0 & -0.8 & -1.8 \\
\hline & $\mathrm{SiSe}_{2}$ & -0.6 & -1.7 & -2.3 \\
\hline $\mathrm{Ge}(1 \mathrm{~s})$ in $\mathrm{GeO}_{2}$ & $\mathrm{GeS}_{2}$ & -0.9 & -0.5 & -1.5 \\
\hline & $\mathrm{GeSe}_{2}$ & -0.7 & -1.2 & -1.9 \\
\hline
\end{tabular}




\section{References}

${ }^{1}$ S. Hüfner, Photoelectron Spectroscopy: Principles and Applications, Springer Series in Solid-State Sciences, Springer-Verlag Berlin Heidelberg GmbH, 2003.

${ }^{2}$ P. Van der Heide, X-ray Photoelectron Spectroscopy. An Introduction to Principles and Practices, John Wiley \& Sons, Inc., Hoboken, New Jersey, 2012.

${ }^{3}$ K. Siegbahn, C. Nordling, A. Falhman, R. Nordberg, K. Hamrin, J. Hedman, G. Johansson, T. Bergmark, S. E. Karlsson, I. Lindgren and B. Lindberg, ESCA-Atomic, Molecular and Solid State Structure Studied by Means of Electron Spectroscopy, Alwmvist and Wiksells Boktryckeri AB, Uppsala, Sweden, 1967.

${ }^{4}$ K. Siegbahn, C. Nordling, G. Johansson, J. Hedman, P. F. Hedén, K. Hamrin, U. Gelius, T. Bergmark, L. O. Werme, R. Manne and Y. Baer, ESCA-Applied to Free Molecules, North-Holland, Amsterdam, 1969.

${ }^{5}$ P. S. Bagus, E. S. Ilton and C. J. Nelin, Catal. Lett., 2018, 148, 1785.

${ }^{6}$ P. S. Bagus, E. S. Ilton and C. J. Nelin, Surf. Sci. Rep., 2013, 68, 273

${ }^{7}$ F. Viñes, C. Sousa and F. Illas, Phys. Chem. Chem. Phys., 2018, 20, 8403

${ }^{8}$ E. Sokolowski, C. Nordling and K. Siegbahn, Phys. Rev., 1958, 110, 776.

${ }^{9}$ P. S. Bagus, F. Illas, G. Pacchioni and F. Parmigiani, J. Electr. Spectrosc. Relat. Phenom., 1999, 100, 215.

${ }^{10}$ N. Pueyo Bellafont, F. Viñes and F. Illas, J. Chem. Theo. Comput., 2016, 12, 324.

${ }^{11}$ J. P. Perdew, K. Burke and M. Ernzerhof, Phys. Rev. Lett., 1996, 77, 3865.

${ }^{12}$ J. P. Perdew, A. Ruzsinszky, G. I. Csonka, L. A. Constantin and J. Sun, Phys. Rev. Lett., 2009, 103, 026403.

${ }^{13}$ N. Pueyo Bellafont, P. S. Bagus and F. Illas, J. Chem. Phys., 2015, 142, 214102.

${ }^{14}$ P.S. Bagus, D. Coolbaugh, S.P. Kowalczyk, G. Pacchioni and F. Parmigiani, J. Electron. Spectry., 1990, 51, 69.

${ }^{15}$ G. Pacchioni, C. Sousa, F. Illas, F. Parmigiani and P.S. Bagus, Phys. Rev. B, 1993, 48, 11573.

${ }^{16}$ P. S. Bagus, G. Pacchioni, C. Sousa, T. Minerva and F. Parmigiani, Chem. Phys. Lett., 1992, 196, 641.

${ }^{17}$ P. S. Bagus, C. R. Brundle, F. Illas, F. Parmigiani and G. Polzonetti, Phys. Rev. B, 1991, 44, 9025.

${ }^{18}$ P. S. Bagus, F. Illas, J. Casanovas and J. M. Jiménez-Mateos, J. Elec. Spec. Related Phenomena, 1997, 83, 151.

${ }^{19}$ P. S. Bagus and H. F. Schaefer, J. Chem. Phys., 1971, 55, 1474.

${ }^{20}$ F. Illas, M. Merchan, M. Pelissier and J.P. Malrieu, Chem. Phys., 1986, 107, 361.

${ }^{21}$ J. Tirado-Rives and William L. Jorgensen, J. Chem. Theory and Comput., 2008, 4, 297.

${ }^{22}$ M. Bühl, and H. Kabrede, J. Chem. Theory and Comput., 2006, 2, 1282.

${ }^{23}$ B. O. Roos, P. R. Taylor, and P. E. M. Siegbahn, Chem. Phys., 1980, 48, 157. 
${ }^{24}$ P. S. Bagus, Phys. Rev., 1965, 139, A619.

${ }^{25}$ M. W. Schmidt, K. K. Baldridge, J. A. Boatz, S. T. Elbert, M. S. Gordon, H. S. J. Jensen, N. Koseki, K. Matsunaga, A. Nguyen, S. Su, T. L. Windus, M. J. Dupuis and A. Montgomery, J. Comput. Chem., 1993, 14, 1347.

${ }^{26}$ M.S. Gordon and M.W. Schmidt, Theory and Applications of Computational Chemistry: the first forty years, Elsevier, Amsterdam, 2005.

${ }^{27}$ N. Pueyo Bellafont, P. S. Bagus, C. Sousa and F. Illas, J. Chem. Phys., 2017, 147, 024106.

${ }^{28}$ F. Aquilante, L. De Vico, N. Ferré, G. Ghigo, P.-Å. Malmqvist, P. Neogrády, T.B. Pedersen, M. Pitonak, M. Reiher, B.O. Roos, L. Serrano-Andrés, M. Urban, V. Veryazov and R. Lindh, J. Comput.

Chem., 2010, 31, 224.

${ }^{29}$ T.H. Dunning, Jr. J. Chem. Phys., 1989, 90, 1007.

${ }^{30}$ D.E. Woon and T.H. Dunning, Jr. J. Chem. Phys., 1993, 98, 1358.

${ }^{31}$ A.K. Wilson, D.E. Woon, K.A. Peterson and T.H. Dunning, Jr. J. Chem. Phys., 1999, 110, 7667.

${ }^{32} \mathrm{https}: / /$ bse.pnl.gov/bse/portal

${ }^{33}$ Schafer, H. Horn and R. Ahlrichs, J. Chem. Phys., 1992, 97, 2571.

${ }^{34}$ J. M. Tao, J. P. Perdew, V. N. Staroverov and G. E. Scuseria, Phys. Rev. Lett., 2003, 91, 146401.

${ }^{35}$ P. S. Bagus, C. Sousa and F. Illas, J. Chem. Phys., 2016, 145, 144303.

${ }^{36}$ N. Pueyo Bellafont, F. Illas and P. S. Bagus, Phys. Chem. Chem. Phys., 2015, 17, 4015. 